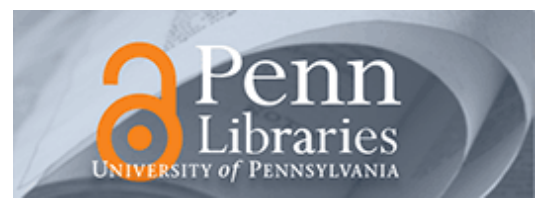

University of Pennsylvania ScholarlyCommons

December 2007

\title{
One and Two Port Piezoelectric Higher Order Contour-Mode MEMS Resonators for Mechanical Signal Processing
}

Gianluca Piazza

University of Pennsylvania, piazza@seas.upenn.edu

Follow this and additional works at: https://repository.upenn.edu/ese_papers

\section{Recommended Citation}

Gianluca Piazza, "One and Two Port Piezoelectric Higher Order Contour-Mode MEMS Resonators for Mechanical Signal Processing", . December 2007.

Postprint version. Published in Solid State Electronics, Volume 51, Issues 11-12, December 2007, pages 1596-1608. Special Issue: Papers Selected from the 36th European Solid-State Device Research Conference - ESSDERC'06.

Publisher URL: http://dx.doi.org/10.1016/j.sse.2007.09.037

This paper is posted at ScholarlyCommons. https://repository.upenn.edu/ese_papers/320

For more information, please contact repository@pobox.upenn.edu. 


\title{
One and Two Port Piezoelectric Higher Order Contour-Mode MEMS Resonators for Mechanical Signal Processing
}

\author{
Abstract \\ This paper reports on the design, fabrication and testing of novel one and two port piezoelectric higher \\ order contour-mode MEMS resonators that can be employed in RF wireless communications as \\ frequency reference elements or arranged in arrays to form banks of multi-frequency filters. The paper \\ offers a comparison of one and two port resonant devices exhibiting frequencies approximately ranging \\ from 200 to $800 \mathrm{MHz}$, quality factor of few thousands (1000-2500) and motional resistances ranging \\ from 25 to $1000 \Omega$. Fundamental advantages and limitations of each solution are discussed. The reported \\ experimental results focus on the response of a higher order one port resonator under different \\ environmental conditions and a new class of two port contour resonators for narrow band filtering \\ applications. Furthermore, an overview of novel frequency synthesis schemes that can be enabled by \\ these contour-mode resonators is briefly presented.

\section{Keywords} \\ RF MEMS, piezoelectric resonator, contour-mode resonators, aluminum nitride

\section{Comments} \\ Postprint version. Published in Solid State Electronics, Volume 51, Issues 11-12, December 2007, pages \\ 1596-1608. \\ Special Issue: Papers Selected from the 36th European Solid-State Device Research Conference - \\ ESSDERC'06. \\ Publisher URL: http://dx.doi.org/10.1016/j.sse.2007.09.037
}




\title{
One and Two Port Piezoelectric Higher Order Contour-Mode MEMS Resonators
} for Mechanical Signal Processing

\author{
Gianluca Piazza $^{1}$, Philip J. Stephanou ${ }^{2}$ and Albert P. Pisano ${ }^{3}$ \\ ${ }^{1}$ Department of Electrical and Systems Engineering, University of Pennsylvania, \\ Philadelphia, PA, 19104 \\ 2 Harmonic Devices, Inc., Berkeley, CA, 94720 \\ ${ }^{3}$ Berkeley Sensor and Actuator Center, University of California at Berkeley, \\ Berkeley, CA, 94720
}

\author{
Corresponding Author: \\ Dr. Gianluca Piazza, \\ University of Pennsylvania \\ 200 S. $33^{\text {rd }}$ Street, Philadelphia, PA, 19104 \\ Room: 360 GRW \\ Tel: 215-573-2812 \\ Fax: 215-573-2068 \\ Email: piazza@seas.upenn.edu
}

\begin{abstract}
This paper reports on the design, fabrication and testing of novel one and two port piezoelectric higher order contour-mode MEMS resonators that can be employed in RF wireless communications as frequency reference elements or arranged in arrays to form banks of multi-frequency filters. The paper offers a comparison of one and two port resonant devices exhibiting frequencies approximately ranging from 200 to $800 \mathrm{MHz}$, quality factor of few thousands $(1,000-2,500)$ and motional resistances ranging from 25 to $1000 \Omega$. Fundamental advantages and limitations of each solution are discussed. The reported experimental results focus on the response of a higher order one port resonator under different environmental conditions and a new class of two port contour resonators for narrow band filtering applications. Furthermore, an overview of novel frequency synthesis schemes that can be enabled by these contour-mode resonators is briefly presented.
\end{abstract}


KEYWORDS: RF MEMS, piezoelectric resonator, contour-mode resonators, aluminum nitride.

\section{INTRODUCTION}

Recent advances in surface micromachining techniques have enabled the realization of miniaturized and high quality factor bulk acoustic resonators that can be integrated with state-of-the-art CMOS electronics [1-8]. Amongst these MEMS devices, a new class of resonators, dubbed contour mode because of their in-plane mode of vibration, has received large attention due to its ability to provide multiple frequency devices on the same silicon substrate. These laterally vibrating microstructures not only provide the advantages of compact size, low power consumption and compatibility with high yield mass producible components, but can also enable paradigm-shifting solutions for simpler frequency synthesizers and transceivers. For example, direct frequency synthesis without the need of complicated phase locked loops will be possible in spread spectrum communication systems via multi-frequency narrowband filter banks or high $Q$ resonators.

Electrostatic and piezoelectric transduction mechanisms have emerged [3, 4] as the preeminent techniques for driving and sensing resonant vibrations in micromechanical structures. Electrostatically-transduced resonators have demonstrated extremely high quality factors and high frequencies (up to $\mathrm{GHz}$ by employing overtones), but suffer from large motional impedances that make their interface with $50 \Omega$ RF systems troublesome. 
Recent research activities $[9,10]$ have demonstrated that lower impedances (100 $\Omega$ to $10 \mathrm{k} \Omega$ ) can be obtained via dielectric transduction by filling the actuation gap with solid high-k dielectrics. Although very promising, this technology is still unproven and fundamentally suffers from large intrinsic capacitances that mask the resonator response at high frequencies or complicate their interface with standard circuitry.

Piezoelectric actuation in aluminum nitride contour-mode resonators has been proven as a superior technology $[6,11-14]$, capable of intrinsically providing low motional resistance (25 to $1000 \Omega$ ) while maintaining high quality factors $(1,000-2,500)$ and reasonable reactance values that ease their interface with state-of-the-art circuitry. This paper reports on the design and experimental results of one and two port implementations of piezoelectric contour-mode resonators and describes advantages and disadvantages of each solution. Furthermore, experimental results for a novel one port higher order contour-mode solution employed to reach frequencies as high as $803 \mathrm{MHz}$ and a new class of two port resonators for the implementation of narrowband filter banks are presented. 


\section{RESONATOR DESIGN}

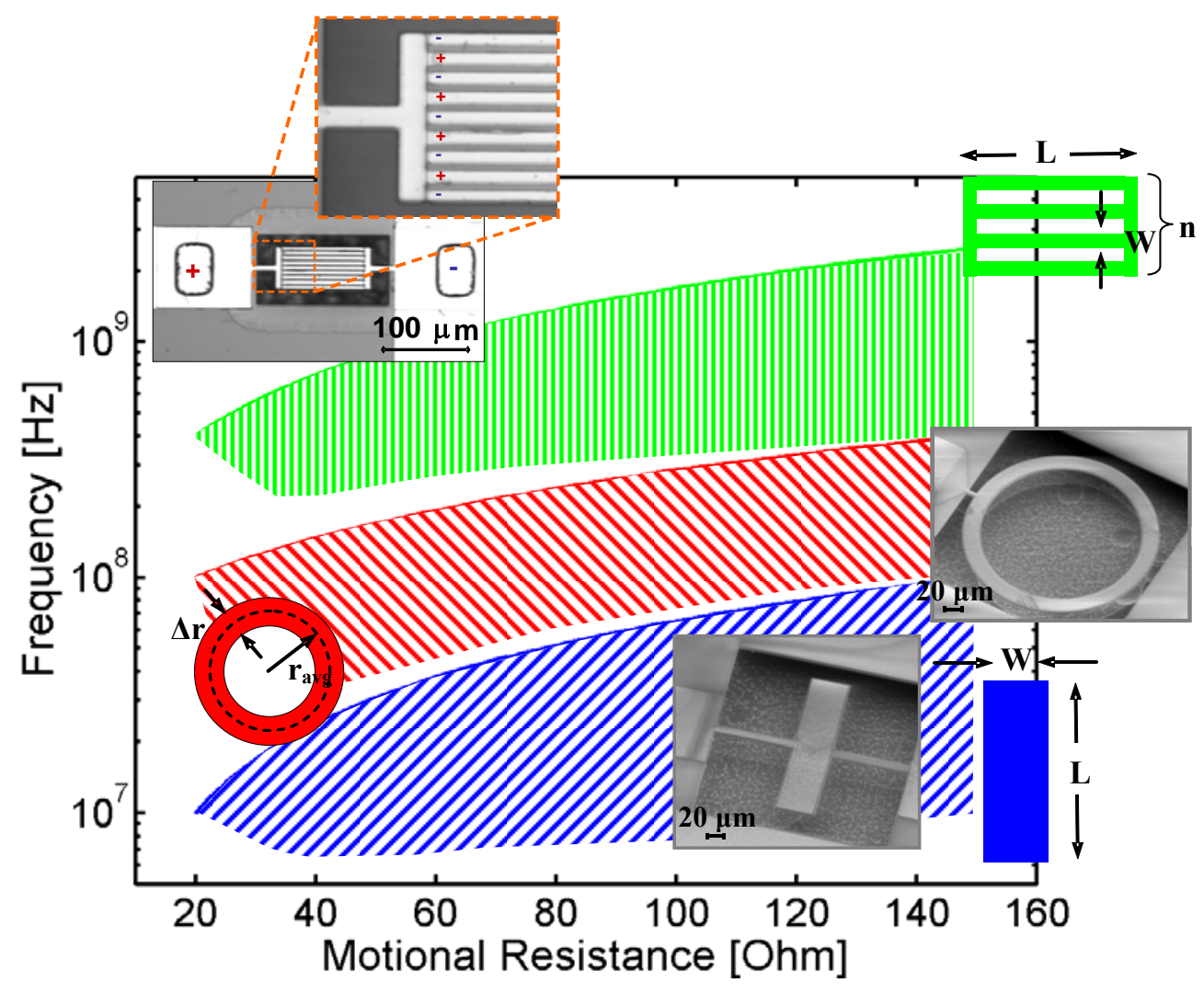

Figure 1: Design space (frequency versus equivalent motional resistance) for contourmode AlN resonators

Contour mode of vibrations can be excited in c-axis oriented aluminum nitride films via the $d_{3 l}$ piezoelectric coefficient. By applying an electric field across the film sandwiched between a top and bottom electrode, the MEMS structure tends to expand laterally and can be excited in resonant vibrations whose frequency is set by the in-plane dimensions of the device [6]. The most promising structures to obtain high $Q$ and high frequency of operations are rings and rectangular plates as shown in Figure 1. The frequency of vibration is generally set by the width of the structure, whereas the second dimension can be employed to control the equivalent motional resistance and static capacitance of the device. This is an additional degree of freedom that a disk structure does not offer. As 
shown in Figure 1 and according to experimental results, manufacturing considerations and theoretical observations (structural rigidity):

- $\quad$ the rectangular plate geometry [6] can be effectively employed from 10 to approximately $200 \mathrm{MHz}$;

- $\quad$ the ring geometry [12] can be adopted between 100 and $450 \mathrm{MHz}$;

- $\quad$ the higher order contour-mode rectangular geometry [15] can be used between 200 and 2,000 MHz.

Although based on preliminary results and subject to improvements through future research, these guidelines offer a good prospective of the status and range of applicability of the AlN contour-mode technology.

The equivalent circuit representation for a piezoelectric transducer is a simplified version of the Mason's model as shown in Figure 2 [16].

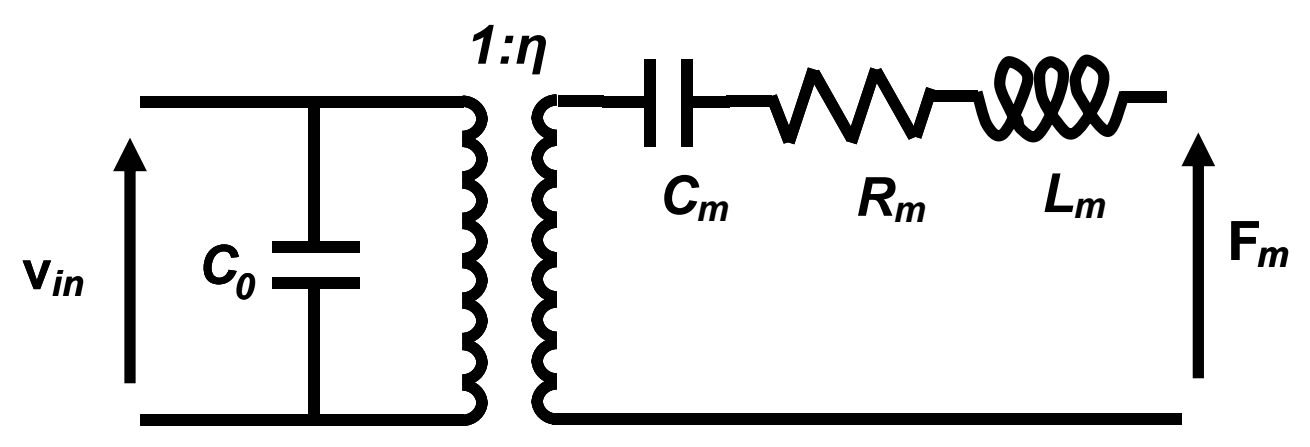

Figure 2: Conventional Mason lumped circuit model for a piezoelectric transducer.

This model will be employed in the following sections to describe one and two port devices and analyze their fundamental characteristics. As shown in Figure 2, the transducer can be modeled by: an intrinsic capacitance, $C_{0}$, representing the physical capacitance of the electroded part of the piezoelectric device; a transformer, whose turn ratio, $\eta$, represents the conversion between electrical and mechanical variables at a 
specific location of the device (generally the point of maximum displacement); and motional capacitance, $C_{m}$, resistance, $R_{m}$, and inductance, $L_{m}$, representing the mechanical variables of the MEMS resonators, and being associated with compliance $\left(1 / k_{e q}\right)$, damping $\left(c_{e q}\right)$ and mass $\left(m_{e q}\right)$, respectively (see Table I). More specifically:

$$
\begin{array}{ccc}
C_{0}=\varepsilon_{P} & \text { Electroded Area } & \eta=\frac{F}{V}=\frac{I}{v} \\
C_{m}=\frac{1}{k_{e q}} & L_{m}=c_{e q} &
\end{array}
$$

where $\varepsilon_{P}$, is the permittivity of the piezoelectric material, and $F, V, I$, and $v$ are defined in Table I.

Table I: Mapping between mechanical and electrical variable used to derive the equivalent electrical circuit of Figure 2. The arrow emphasizes the fact that it is a mapping process and proportionality factors are not explicitly indicated.

\begin{tabular}{||c|c||}
\hline MECHANICAL VARIABLE & ELECTRICAL ANALOGUE \\
\hline Force $(F)$ & Voltage $(V)$ \\
\hline Velocity $(v)$ & Current $(I)$ \\
\hline Compliance $\left(1 / k_{e q}\right)$ & Capacitance $\left(C_{m}\right)$ \\
\hline Damping $\left(c_{e q}\right)$ & Resistance $\left(R_{m}\right)$ \\
\hline Mass $\left(m_{e q}\right)$ & Inductance $\left(L_{m}\right)$ \\
\hline
\end{tabular}

\section{A. One Port Resonators}

A one port resonator is a device for which the simplified model of Figure 2 is terminated in a short, representing, in the acoustic domain, a perfectly reflective boundary such as air. In one port configurations, the static capacitance of the device appears in parallel 
with the motional components of the resonator and its value depends, if fringing fields are neglected, only on the electroded area.

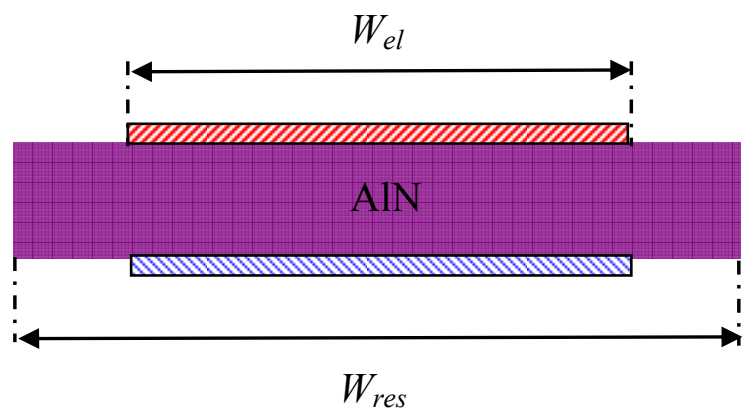

(a)

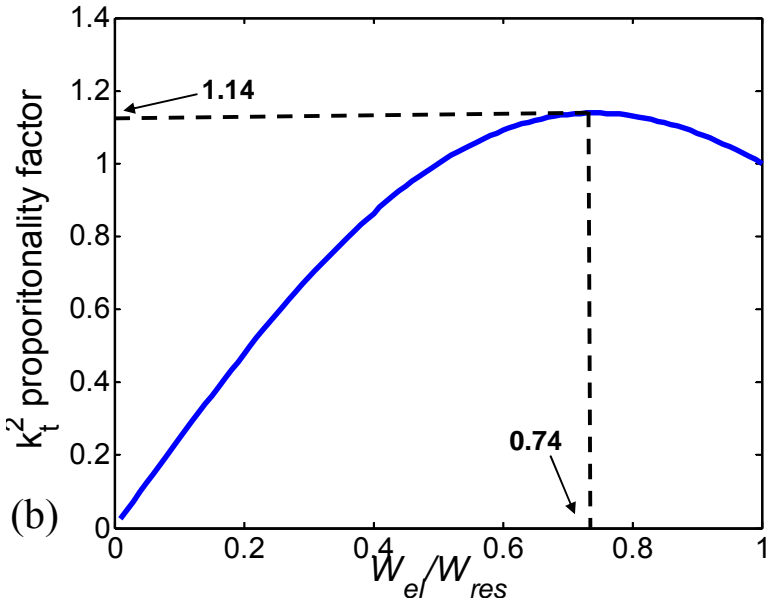

Figure 3: (a) cross-sectional view of partially electroded plate or ring resonator showing the fundamental parameters in Equation (2). (b) Relative change (proportionality factor of Equation (2)) in $k_{t}^{2}$ with respect to percentage of electrode to resonator width coverage.

Particular attention should be placed in patterning the electrodes which set not only the motional impedance of the device, but also its electromechanical coupling coefficients, $k_{t}^{2}$, and affect selective excitation of mechanical modes. For example, a completely electroded surface, such as the one adopted for plates and rings [6], yields the lowest possible motional resistance and higher electromechanical strength for the fundamental mode over spurious inharmonic resonances, but theoretically limits $k_{t}^{2}$ to about $88 \%$ of its maximum value (Fig. 3b). In fact, for one-dimensional analysis of the vibrations of a partially electroded plate or ring (Fig. 3a), $k_{t}^{2}$ can be found to be [17]:

$$
k_{t}^{2} \propto \frac{W_{\text {res }}}{W_{e l}}\left[\sin \left(\frac{\pi}{2} \frac{W_{e l}}{W_{\text {res }}}\right)\right]^{2}
$$

where $W_{r e s}$ and $W_{e l}$ are the resonator and electrode widths as shown in Figure 3. 
As Figure 3 shows, maximum $k_{t}^{2}$ can be achieved by making the electrodes approximately $3 / 4$ as long as the bar or strip. This is a trade-off that the designer needs to consider when laying out the structure.

In order to obtain high manufacturing yield, the device center frequency needs to be set with accuracy within $0.1 \%(1000 \mathrm{ppm})$, before any post-processing trimming is executed. Film thickness has only a second order effect on frequency and the contourmode device sensitivity to thickness variations is at least 10x lower than FBAR technology [6]. Sputtering deposition tools can easily provide $0.5 \%$ uniformity across $200 \mathrm{~mm}$ wafers, therefore satisfying frequency requirements in the thickness direction for contour-mode resonator (it remains a challenge for FBAR for which absolute thickness control is more demanding). The lateral dimensions of the structure have instead a direct impact on the frequency of the contour-mode device (as the thickness does in FBAR resonators). Standard lithography tools generally have tolerances in the order of $5 \%$ of their minimum feature size. This means that $0.1 \%$ accuracy can be easily obtained for frequencies as high as $200 \mathrm{MHz}$ using old generation lithography tools and having a minimum feature size of $0.5 \mu \mathrm{m}$. Lithographic definitions of lateral features for high frequency devices can pose a challenge for reliable and reproducible manufacturing of contour mode resonators.

In order to desensitize the resonator center frequency from the definition of absolute lateral dimensions and simultaneously maintain adequate structural rigidity at high frequencies, a new contour-mode device based on selective excitation of higher order modes of vibration in rectangular plates has been devised [15]. Two excitation schemes have been proposed and are based on thickness field excitation (TFE) and lateral field 
excitation (LFE). As shown in Figure 4, adjacent strips of the rectangular plate are excited out of phase with respect to each other by opposite direction electric fields generated either by opposing (TFE) or by coplanar (LFE) electrodes. High frequency contour modes of vibration (as high as $\mathrm{GHz}$ ) can now be selectively transduced in large plates (100x51 $\mu \mathrm{m}$ for the $803 \mathrm{MHz}$ rectangular plate shown here). Electrode periodicity and plate absolute dimensions now set the center frequency of the device and dramatically relax manufacturing constraints in a manner similar to SAW devices [18]. Furthermore, the number of rectangular strips can be prescribed at the mask layout level to arbitrarily scale the motional resistance and static capacitance of the device.

The same design trade-off between $k_{t}^{2}, R_{m}$ and spurious modes excitation described for the fundamental mode device apply as well to the higher order mode design.

The equivalent electromechanical parameters of the higher order contour-mode resonator using a TFE configuration can be approximately found using the same techniques developed for a fundamental mode resonator [6]. In the case of a higher order mode resonator each rectangular strip that forms the device can be considered as a fundamental mode resonator in parallel with the other $n$-1 resonators (where $n$ is the total number of parallel electroded strips). Therefore the equivalent electromechanical parameters shown in Figure 2 can be approximated by: 


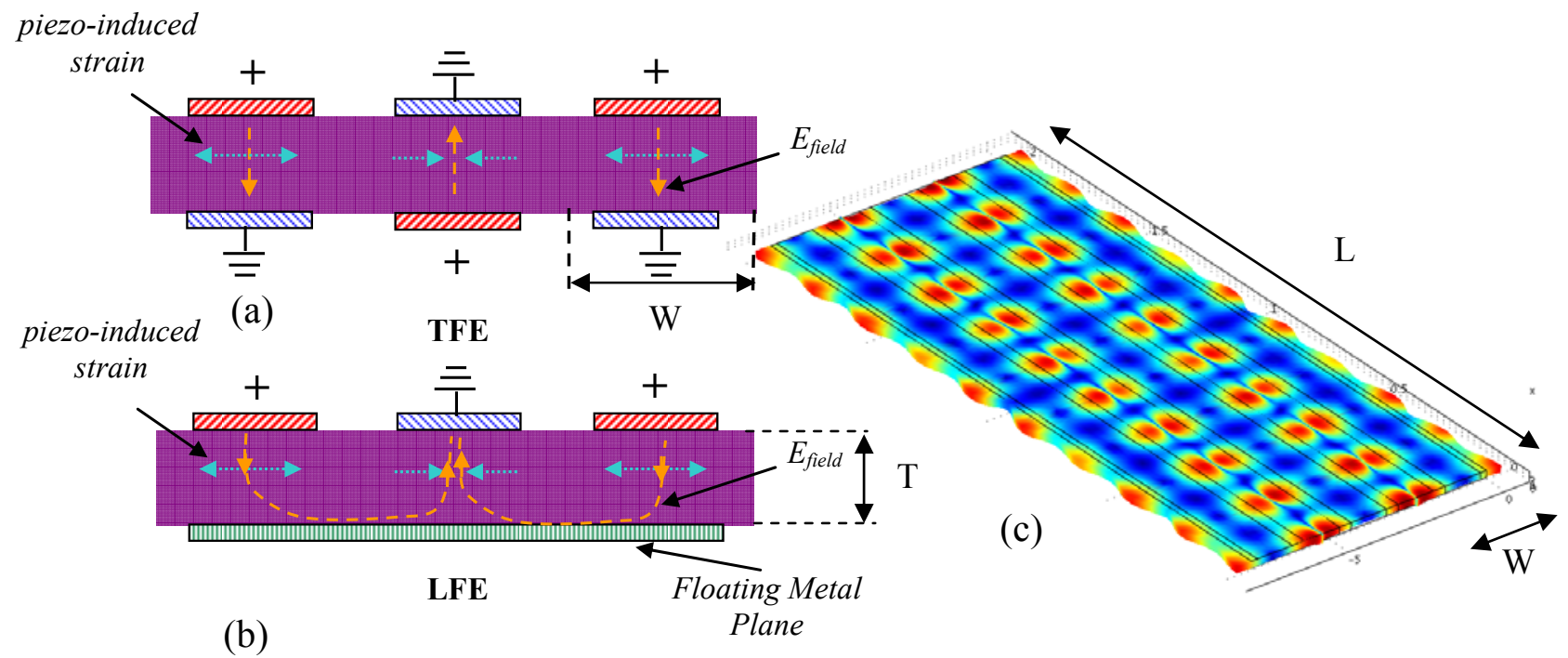

Figure 4: Schematic representation of the excitation scheme used to induce higher order contour modes in rectangular plates. (a) TFE is characterized by opposing electrodes, whereas (b) LFE makes use of coplanar electrodes. In the LFE implementation a floating metal plane was included to improve the deposition of AIN films and offer better confinement of the electric field within the entire thickness of the AIN resonator. (c) COMSOL simulation shows a three dimensional example of the mode of vibration of higher order contour-mode resonators for which five parallel electrodes were considered.

$$
\begin{gathered}
C_{0}=n \cdot \varepsilon_{P} \frac{W L}{T} \quad \eta=2 d_{31} E_{e q} L \\
C_{m}=n \cdot \frac{2}{\pi^{2}} \frac{W}{L T} \frac{1}{E_{e q}} \quad R_{m}=\frac{1}{n} \frac{\pi}{2} T L \frac{\sqrt{E_{e q} \rho_{e q}}}{Q} \quad L_{m}=\frac{1}{n} \cdot \frac{\rho_{e q}}{2} L W T
\end{gathered}
$$

where $W, L$ and $T$ refer to the dimensions of a single rectangular strip, $E_{e q}$ and $\rho_{e q}$ are the equivalent modulus of elasticity and mass density of the electroded AIN plate [6].

For a higher order contour-mode device using LFE excitation the equivalent parameters cannot be accurately approximated by closed form analytical expressions because the electromechanical coupling, $\eta$, is a strong function of the electric field distribution which depends on the electrode spacing and film thickness. Equivalent parameters can be 
derived by FEM simulations. For example, Figure 5 shows a comparison between the admittance curves for two higher order contour mode resonators excited in TFE and LFE modes, each having $n=7$. The frequency shift is a consequence of adding the bottom Pt layer in the LFE simulations, whereas Pt electrodes were not included in the TFE simulation. As shown in Figure 5 the LFE resonator is characterized by lower coupling efficiency, a smaller equivalent $C_{0}$ and a larger motional resistance than the TFE device.

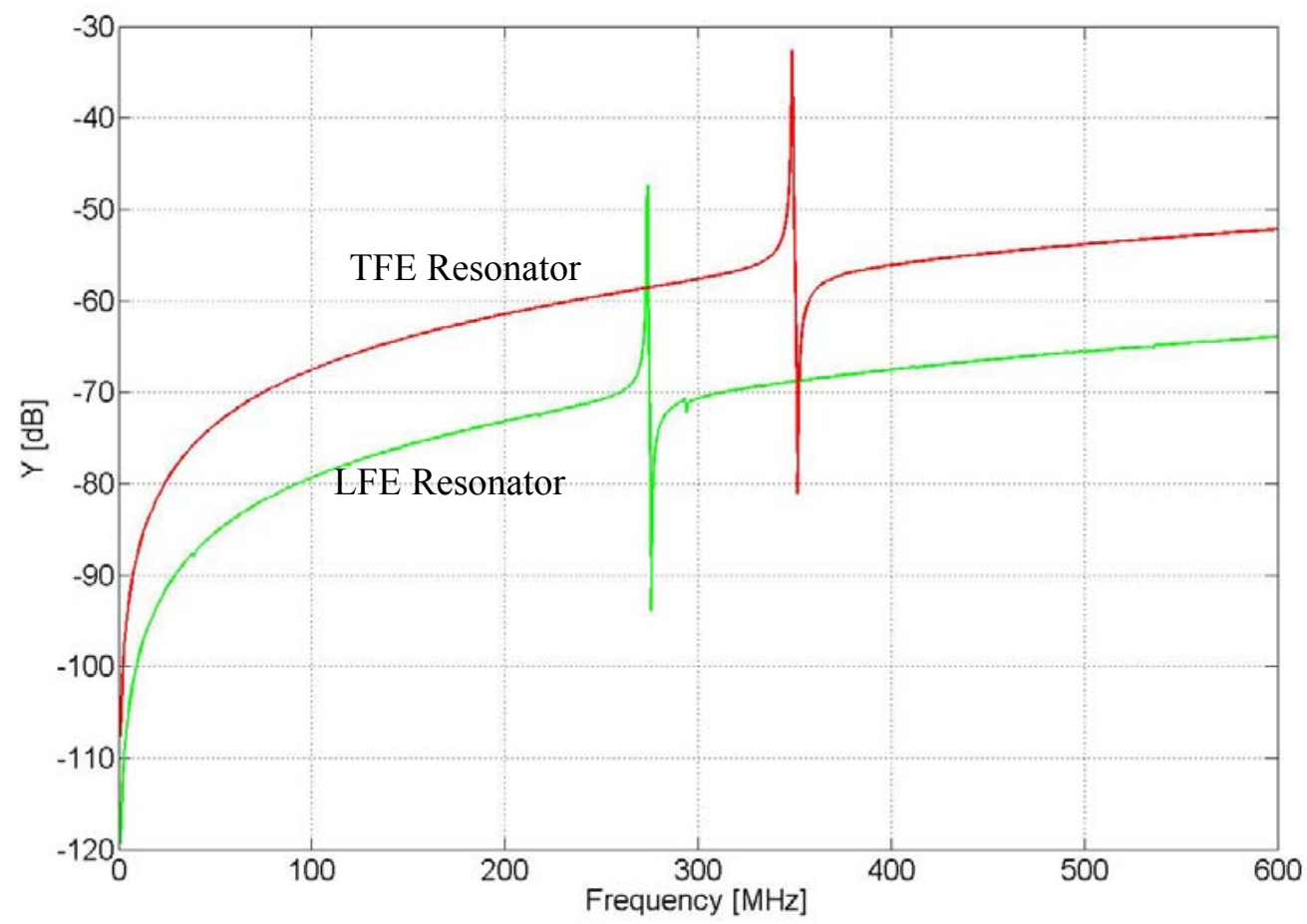

\begin{tabular}{|l|c|c|c|c|}
\hline Resonator & $k_{t}^{2}[\%]$ & $C_{0}[\mathbf{f F}]$ & $R_{m}[\Omega]$ & $C_{m}[\mathbf{f F}]$ \\
\hline LFE resonator & 1.79 & 170 & 230.9 & 0.0442 \\
\hline TFE resonator & 2 & 665 & 41.2 & 0.2156 \\
\hline
\end{tabular}

Figure 5: Equivalent admittance curves and fundamental electromechanical parameters of two rectangular plate resonators using TFE and LFE. The plates have $n=7$ and overall dimensions of $105 \times 200 \mu \mathrm{m}$. The results are obtained using COMSOL two dimensional FEM analysis 


\section{B. Two Port Resonators}

A two port resonator can be represented by two cascaded networks of the type depicted in

Figure 2. Ideally, it can be modeled as two resonators coupled at an infinitely stiff location. This implementation is generally encountered in macroscale resonant piezoelectric transformers. A clear advantage of this solution over a one port counterpart is the electrical isolation of the input and output terminals that facilitates the transduction of the resonators at higher frequencies. This, in fact, is the only demonstrated implementation for electrostatically-driven resonators above few MHz. Two possible embodiments for two port piezoelectric resonators have already been demonstrated [19, 20] (Fig. 6). In Figure 6a the two port configuration is realized by placing the electrodes adjacent to each others in the lateral dimensions [19]. Another topology consists of stacking the transducers on top of one another (Fig. 6b) [20]. This second implementation is more effective since it matches the motional resistance of a one port device for a given layout area and improves the mechanical coupling into the fundamental mode by effectively driving the device over the entire top surface. Furthermore, this second solution limits the input to output capacitive feedthrough to the substrate by eliminating coupling through the AlN film that is present between the driving and sensing electrodes in the first configuration. The two port stacked topology was implemented for both rectangular and ring geometries.

The work presented in this paper focus on higher order contour-mode resonators. Figure $6 \mathrm{c}$ shows a possible embodiment of a two port topology in a higher order contourmode device. In this case we defined the two ports of the resonator by placing the electrodes on separate halves of the device and split them in a direction parallel to the 
acoustic wave. Although this solution is less effective than a stacked topology, it is the most intuitive and easiest way of realizing a two port configuration in higher order contour-mode devices.

(a)

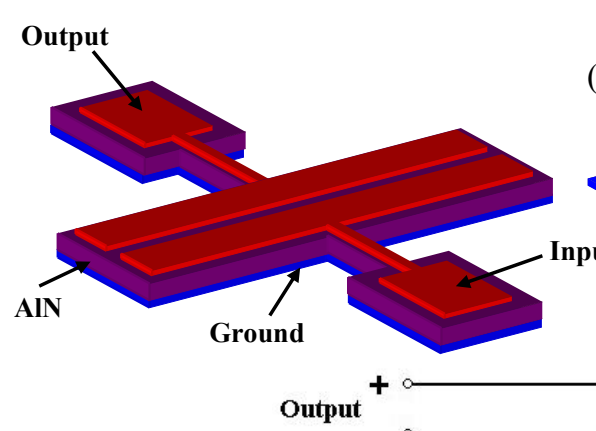

(b)<smiles>[CH]CCCO</smiles>

(c)

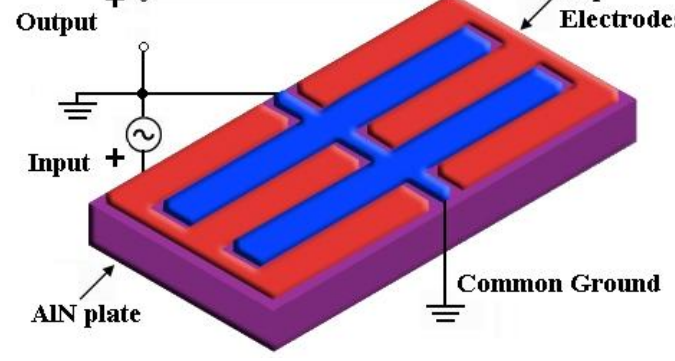

Figure 6: Schematic representation of different implementation of two port contour-mode resonators. (a) Laterally adjacent electrodes constitute the two ports in a fundamental mode resonator. In (b) a stacked configuration is presented. In (c) the two ports are defined in a higher order contour-mode resonator by separating the electrodes along a direction parallel to the acoustic wave in the device. Only the top electrodes are visible in this representation. Bottom electrodes are symmetrically placed at the bottom of the structure to effectively excite higher order contour modes of vibration using a TFE scheme.

A common ground electrode for input and output electrodes is employed and routed via the tethers located in a direction parallel to the acoustic wave in the resonator (Fig. 7). Two more tethers, orthogonal to the direction of the acoustic wave, are used to route the input and output electrical signals. A disadvantage of this design is the number of anchors that support the device. The four anchors (two more with respect to what was used in previous device implementations) constrain the mode of vibration of the device and have a deleterious effect on the quality factor of the device, as proven by the 
experimental results. For this two port higher order contour-mode device the equivalent electromechanical parameters of one-half of the resonator (other half is symmetric with respect to $F_{m}$ ) can be derived in the same manner shown for a one port device and are given by:

$$
\begin{gathered}
C_{0}=n \cdot \varepsilon_{P} \frac{W L}{2 \cdot T} \quad \eta=d_{31} E_{e q} L \\
C_{m}=n \cdot \frac{4}{\pi^{2}} \frac{W}{L T} \frac{1}{E_{e q}} \quad R_{m}=\frac{1}{n} \frac{\pi}{4} T L \frac{\sqrt{E_{e q} \rho}}{Q} \quad L_{m}=\frac{1}{n} \cdot \frac{\rho_{e q}}{4} L W T
\end{gathered}
$$

where $L, W$, and $T$ are the overall dimensions of the structure as shown in Figure 7 .

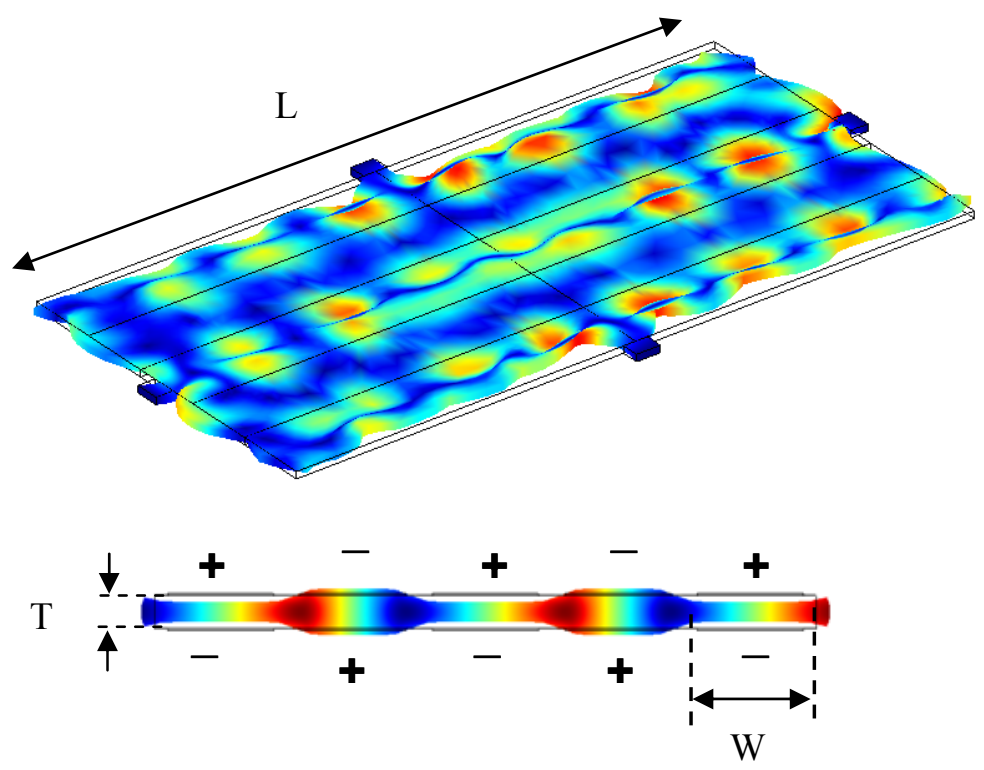

Figure 7: 3D and 2D COMSOL simulations of the mode of vibration of a two port higher order contour-mode resonator.

The need for a common ground or a completely separated set of input and output electrodes limits the number of two port solutions available for higher order contourmode resonators. A stacked topology can also be envisioned if LFE is employed. This scheme is ultimately more complicated to realize and its effectiveness is yet to be proven. 
Whichever implementation is selected, a key characteristic of the two port configuration is that there is either a small value or no direct electrical capacitance between the input and output signals. The device has instead input and output capacitances to ground that can be controlled by the designer. These capacitors can be employed, instead of external off-chip components, as coupling elements for the realization of narrow-band filters [21].

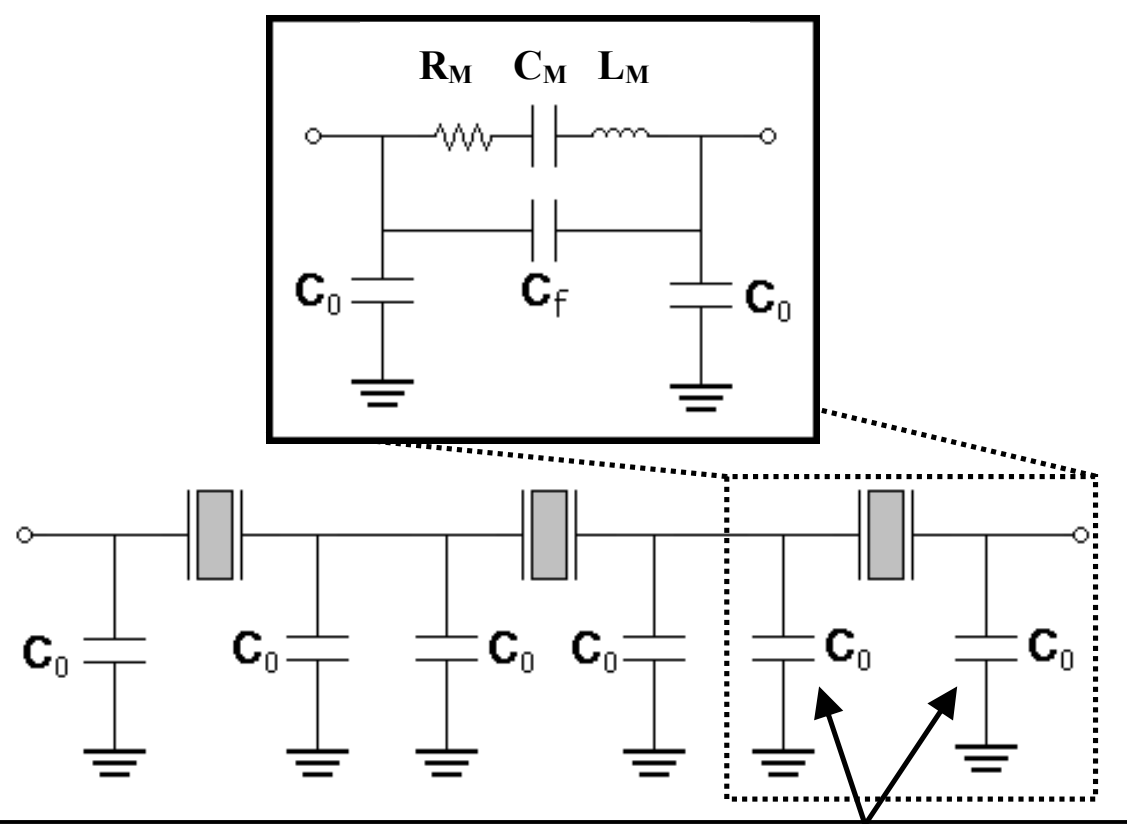

Coupling capacitors are provided by intrinsic capacitance of the resonator

Figure 8: Band pass filter topology based on capacitively-coupled two port resonators. The coupling capacitance, $C_{0}$, is part of the resonator itself. A simplified version of the two port equivalent circuit of a resonator is shown in the inset. In this case the transformer has been eliminated (impedances have been transformed) and a feed through capacitance to account for parasitic components has been added. Capital subscripts are used to identify the transformed motional components of the resonator. The same notation is used throughout the paper.

As schematically shown in Figure 8, a band pass filter can be implemented by electrically cascading two port devices without the need for external capacitors. This filter topology reduces the number of external components required for its realization and especially eliminates the need to define two-frequency resonators as commonly done in ladder 
configurations $[13,22]$. It is therefore clear that this implementation is more amenable to the realization of narrowband filters that can be employed for frequency synthesis. For this filter configuration the $3 \mathrm{~dB}$ bandwidth (BW) depends on the number of cascaded resonators and the electromechanical coupling of the piezoelectric film. For a $3^{\text {rd }}$ order filter it can be approximated by:

$$
B W_{\text {filter }} \approx \frac{3}{8} k_{t}^{2}
$$

\section{DEVICE FABRICATION}

The fabrication process employed for manufacturing the resonant microstructures of this work is based on surface micromachining techniques that are compatible with state-ofthe-art CMOS production systems (Fig. 9). The process employed for this work is the same as described in [6]. The metallization steps are conventional micromachining steps, whereas the etching of AlN and the release of the microstructures could be considered somewhat exotic. In the last decade, etching of III-V compounds and $\mathrm{XeF}_{2}$ release are gaining wider use and understanding and they are considered reliable for high yield and large volume electronic manufacturing.

Figure 10 shows a higher order contour-mode resonator made out of a $1.5 \mu \mathrm{m}$ thick AlN film sandwiched between $200 \mathrm{~nm}$ thick Pt electrodes. The inset highlights the periodicity of the alternating electrodes and its importance in setting the center frequency of the device. It is the period of the electrodes that determines the frequency of the resonant structure, whereas the absolute dimensions of the electrodes and plate have only a second order effect on the center frequency. For example, the frequency sensitivity to changes in the width of the Pt electrodes of a higher order contour-mode resonator with a $6 \mu \mathrm{m}$ pitch 
and $n=9$, AlN thickness of $1.5 \mu \mathrm{m}$ and $200 \mathrm{~nm}$ Pt electrodes is approximately 105 $\mathrm{ppm} / \mathrm{nm}$. A fundamental mode resonator at the same frequency would have shown a frequency sensitivity of almost $210 \mathrm{ppm} / \mathrm{nm}$. A more significant gain in reducing the sensitivity of the resonant frequency to lithographic tolerance can be achieved if $\mathrm{Pt}$ is replaced by Al. With Al electrodes (characterized by a lower mass density than Pt) the sensitivity to lithographic errors shifts from $177 \mathrm{ppm} / \mathrm{nm}$ for a fundamental mode device to approximately $5 \mathrm{ppm} / \mathrm{nm}$ for a higher order contour-mode device having the same dimensions of the previously cited example.

Figure 11 shows a fabricated $3^{\text {rd }}$ order filter realized using two port higher order resonators. This filter was realized in a $2 \mu \mathrm{m}$ AlN film sandwiched between $150 \mathrm{~nm}$ thick Pt electrodes. In this case the mass loading of the Pt was employed to slightly shift the center frequency of adjacent filters to create closely spaced narrow band filters. The resonators forming each of the filters have the same frequency, but each filter has a slightly different center frequency. The shift was obtained by lithographically removing small amounts of $\mathrm{Pt}$ ( 3 and $6 \mu \mathrm{m}$ respectively) from the edge of the fingers of the higher order mode resonators. As shown by the experimental results, a set of three closely frequency spaced filters was successfully fabricated. 


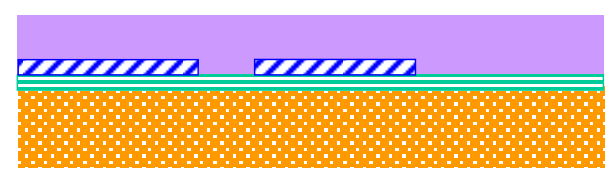

(a)

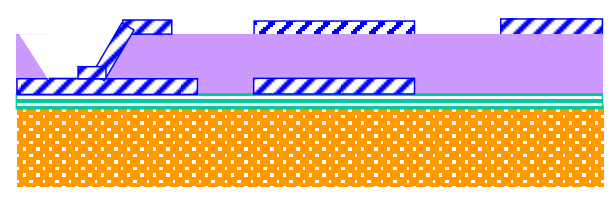

(c)

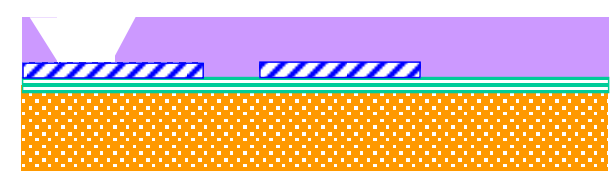

(b)

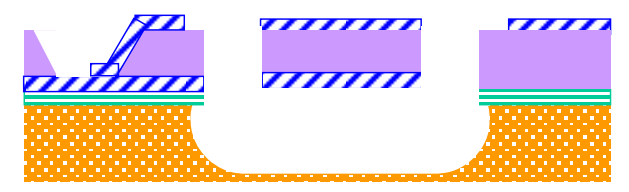

(d)

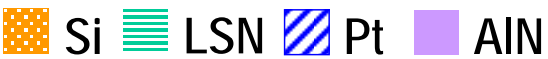

Figure 9: Schematic representation of the fabrication process employed for the making of contour-mode AlN resonators. (a) Low stress nitride (LSN) deposition by LPCVD, followed by Pt patterning by lift-off and AIN sputter deposition; (b) Open via access to bottom Pt electrode through AlN. AlN is wet etched by $160{ }^{\circ} \mathrm{C}_{3} \mathrm{PO}_{4}$; (c) Deposition of top Pt electrode and patterning by lift-off; (d) $\mathrm{Cl}_{2}$-based dry etching of $\mathrm{AlN}$ resonant device and dry release in $\mathrm{XeF}_{2}$.

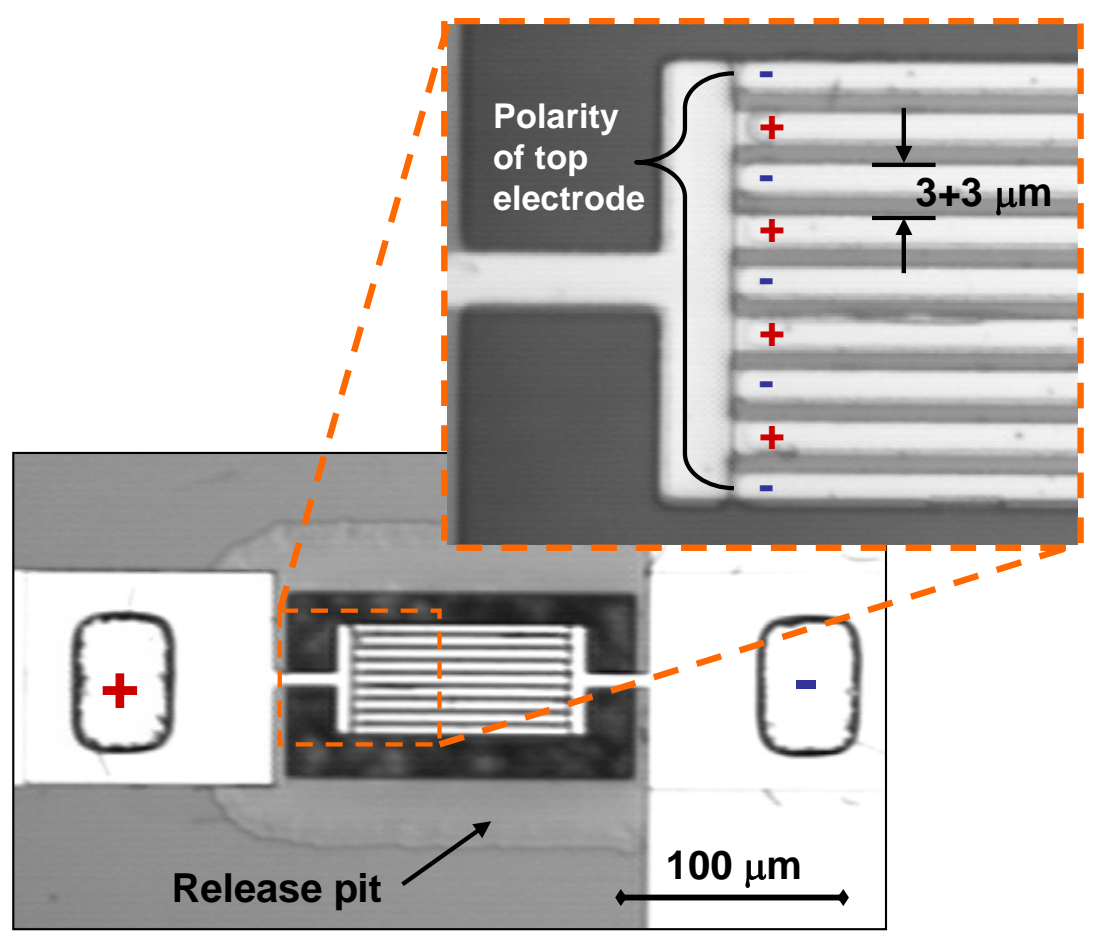

Figure 10: Optical micrograph of micromechanical resonator with selectively patterned half-wavelength electrodes. The polarity of the electric excitation signal alternates between adjacent top and bottom electrode pairs. 


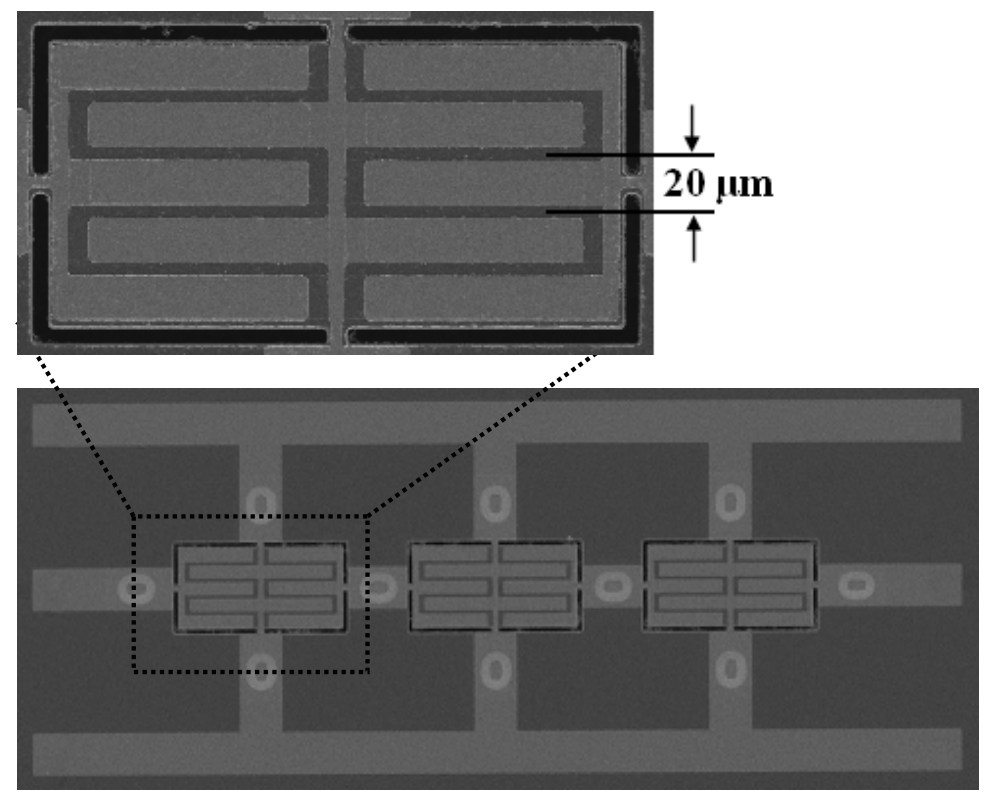

Figure 11: Scanning electron micrograph of a two port higher order contour-mode filter. Inset shows a zoomed in view of the two port resonator. In this case the AlN film is $2 \mu \mathrm{m}$ thick and sandwiched between $150 \mathrm{~nm}$ thick top and bottom Pt electrodes.

\section{EXPERIMENTAL RESULTS}

One and two port resonators were tested in a micromanipulated RF probe station in ambient and cryogenic conditions. A standard Short, Open, Load and Through (SOLT) calibration was performed using a Picoprobe ceramic substrate and the device under test was directly connected to a network analyzer without the need for any external interface electronics.

\section{A. One port Resonators}

Figure 12 shows a plot of the admittance response for the new class of piezoelectric higher order contour-mode MEMS resonators [23]. The AIN plate in this instance measures $51 \times 100 \mu \mathrm{m}$, and the Pt electrodes have a $3 \mu \mathrm{m}$ line width and spacing (Fig. 10). Notice the effective suppression of spurious modes achieved by the specifically 
engineered electrode configuration. This resonator employed a TFE configuration with $n$ $=9$. The resonator shows a $Q$ of approximately 1,300 and an $R_{M}$ of $24 \Omega$ at $803 \mathrm{MHz}$.

The $Q$ value is derived from a direct measurement of the $3 \mathrm{~dB}$ bandwidth of the resonator. This specific device was fabricated on a regular resistivity silicon substrate.
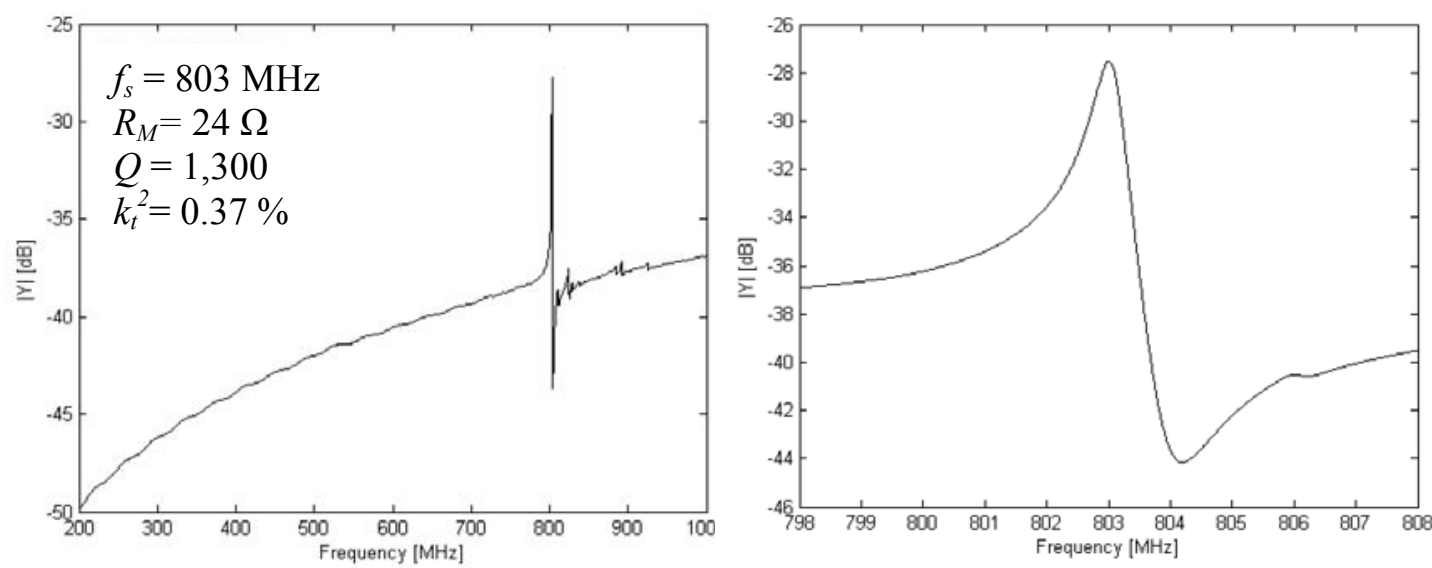

Figure 12: Admittance plots (wide and zoomed in scan) of an $803 \mathrm{MHz}$ higher order contour-mode AlN rsonator.

Higher order contour mode of vibrations have been excited in plates of different dimensions and covering a broad range of frequencies from approximately $200 \mathrm{MHz}$ up to $800 \mathrm{MHz}$. Figure 13 shows the experimental results for a LFE resonator at 216.5 MHz. The device exhibits a $Q$ factor of 1,850 in air and an equivalent motional resistance, $R_{M}$, of $470 \Omega$. This resonator has $n=3$ and is $200 \mu \mathrm{m}$ long and $60 \mu \mathrm{m}$ wide. The responses of both devices of Figures 12 and 13 at resonance and antiresonance are not symmetric, as would instead be predicted by the simple Mason's model. This is because the parasitic effect of the substrate and an additional loss term in the electrode resistance were not taken into account.

The equivalent model of a one port device should be modified such as shown in Figure 14. The series resistance, $R_{s}$, models the electrodes and pads resistance, whereas $R_{L}, C_{L}$, 
$R_{H}$ and $C_{H}$ take into account the complex behavior of the substrate underneath the electrodes and formed by the stack of AlN, low stress nitride (LSN) and high resistivity silicon (for the reported data AlN thickness is $2 \mu \mathrm{m}$, LSN thickness is approximately $300 \mathrm{~nm}$ and the Si has a resistivity larger than $60 \mathrm{k} \Omega \cdot \mathrm{cm}$ ).

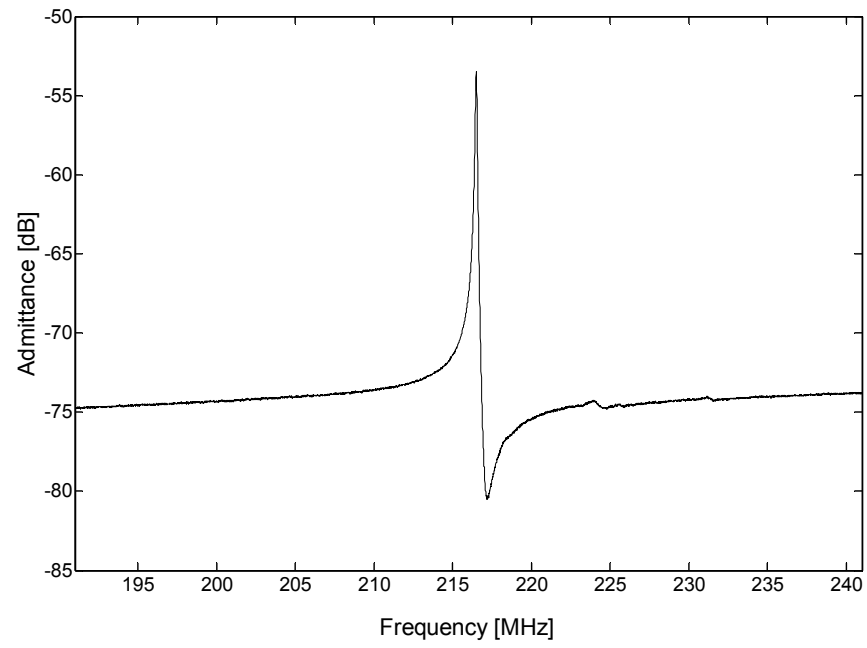

LFE contour-mode resonator $f_{s}=216.5 \mathrm{MHz}$ $R_{M}=470 \Omega$ $Q=1850$ $\mathrm{k}_{\mathrm{t}}^{2}=0.79 \%$

Figure 13: Experimental response of 216.5 MHz LFE higher order contour-mode resonator exhibiting a $Q$ of $1850, R_{M}$ of $470 \Omega$ and $k_{t}^{2}$ of $0.79 \%$.

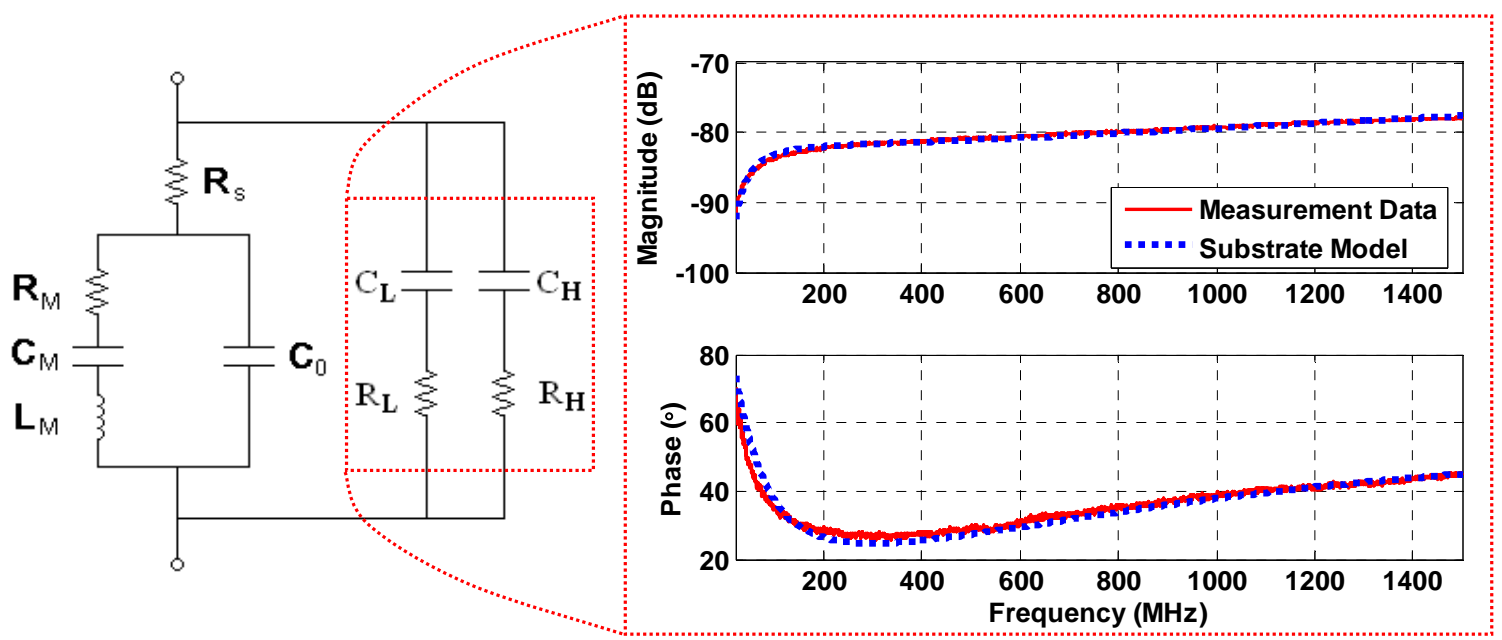

Figure 14: Expanded equivalent model for the one port contour-mode device. The model includes the substrate parasitic components caused by pad and device layout $\left(R_{L}, R_{H}, C_{L}\right.$, $C_{H}$ ) as well as an additional loss term, $R_{S}$, that models the electrical resistance in the electrodes on the device. The plot on the left shows how the substrate model fits very well the measured data for the substrate. 
As shown in Figure 14, the substrate has a frequency dependent behavior and it is better modeled by two series combination of resistor and capacitance in parallel, each representing its characteristics at low (below $300 \mathrm{MHz}$ ) and high (above $300 \mathrm{MHz}$ ) frequencies, respectively.

The equivalent parameters of a higher order contour-mode resonator were extracted taking into account the electrical model of Figure 14 and are presented in Table II. For this resonator the parasitic components were extracted by directly measuring identical pads without a connected device. The results reported in Table II are showing that the series resistance, $R_{s}$, can significantly affect the $Q$ of the resonator, to the point that $30 \%$ loading was recorded in this device. This effect is further verified by testing of the same device at cryogenic temperatures $(78 \mathrm{~K})$. The series resistance drops linearly with the temperature and positively affects the $Q$ of the device. At the same time the $Q$ enhancement cannot be solely explained by the drop in the series resistance. These are the first measurements ever performed at cryogenic temperatures for contour-mode AIN resonators and their explanation is beyond the scope of this paper. As shown in Table II the substrate and pad parasitics (capacitance and resistance) have values that are on par with the device and can significantly impact its response. Ongoing research is trying to mitigate the effect of the substrate on the device response.

\begin{tabular}{|c|c|c|c|c|c|c|c|c|c|c|c|c||}
\hline \multirow{2}{*}{ Device } & $\boldsymbol{T}$ & $\boldsymbol{P}$ & $\boldsymbol{Q}$ & $\boldsymbol{R}_{M}$ & $\boldsymbol{C}_{\boldsymbol{M}}$ & $\boldsymbol{L}_{M}$ & $\boldsymbol{C}_{\boldsymbol{0}}$ & $\boldsymbol{R}_{\boldsymbol{s}}$ & $\boldsymbol{C}_{\boldsymbol{L}}$ & $\boldsymbol{R}_{\boldsymbol{L}}$ & $\boldsymbol{C}_{\boldsymbol{H}}$ & $\boldsymbol{R}_{\boldsymbol{H}}$ \\
\cline { 2 - 14 } & $\mathbf{K}$ & $\mathbf{T o r r}$ & & $\boldsymbol{\Omega}$ & $\mathbf{f F}$ & $\boldsymbol{\mu H}$ & $\mathbf{f F}$ & $\boldsymbol{\Omega}$ & $\mathbf{f F}$ & $\mathbf{k} \boldsymbol{\Omega}$ & $\mathbf{f F}$ & $\mathbf{k} \boldsymbol{\Omega}$ \\
\hline TFE (amb.) & 295 & 760 & 956 & 551 & 1.03 & 284 & 171 & 184 & 241 & 20.57 & 15.17 & 1.22 \\
\hline TFE (cryo.) & 78 & $3.6 \times 10^{-7}$ & 2657 & 198 & 1.03 & 284 & 179 & 53 & 219 & 14.78 & 9.61 & 3.29 \\
\hline
\end{tabular}

Table II: Equivalent electrical parameters of one port higher order contour mode devices extracted from experimental results and fitted to the model presented in Figure 14. Measurements at cryogenics temperatures are also reported. The specific device under test has $n=3$, and overall dimensions of $200 \times 45 \mu \mathrm{m}$. 


\section{B. Two Port Resonators}

The presented two port configuration in higher order contour-mode resonator was employed to successfully implement narrow bandwidth filters. The individual resonators show a $Q$ of approximately 550 and a corresponding motional resistance of $1 \mathrm{k} \Omega$ at $225 \mathrm{MHz}$. The experimental values are showing that this design can be improved, especially if compared to previously demonstrated two port devices $[19,20]$ for which $Q$ comparable to one port devices were reported. Despite the low $Q$ factor, these two port devices were employed to successfully demonstrate narrow band filters.
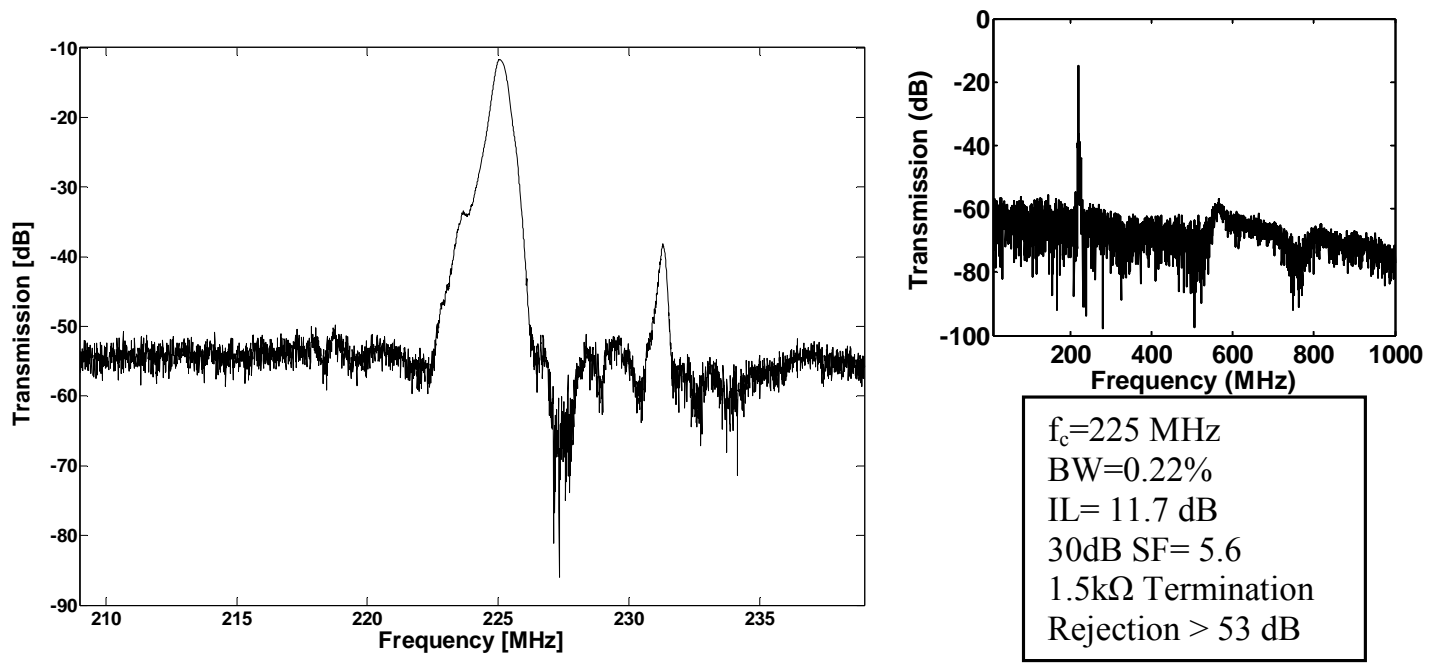

Figure 15: Characteristic response of a $3^{\text {rd }}$ filter implemented using two port higher order contour-mode resonators. In the inset the large span (1 MHz -1,000 MHz) response of the filter is presented showing that spurious responses are well suppressed.

The experimental response of the $3^{\text {rd }}$ order filter of Figure 11 is shown in Figure 15. The filter has a center frequency of $225 \mathrm{MHz}$ and a 3dB bandwidth of $0.22 \%$ with insertion loss (IL) of approximately $11.7 \mathrm{~dB}$ and out-of-band rejection greater than $53 \mathrm{~dB}$. The IL value of these filters is slightly larger than reported for previously demonstrated AlN contour-mode filters [13]. Although on-par with corresponding SAW filters in the same 
frequency range, the relatively large IL is probably due to excessive clamping of the resonator and external electrical loading, which reduce the $Q$ of individual resonators to 550 in air. IL lower than $4 \mathrm{~dB}$ are expected for $Q$ of $2,000$.

Three closely frequency spaced filters were also fabricated and tested. As shown in Figure 16 these filters are the first implementation of narrowband selectable filters. The large IL is again due to the low $Q$ of the device. The key point of this demonstration consists in showing that closely spaced filters can be easily implemented using standard lithographic techniques. The achieved $3 \mathrm{~dB}$ bandwidths are impressively small, in the order of $0.22 \%$, and in line with what would be required for channel selection in WCDMA standards.

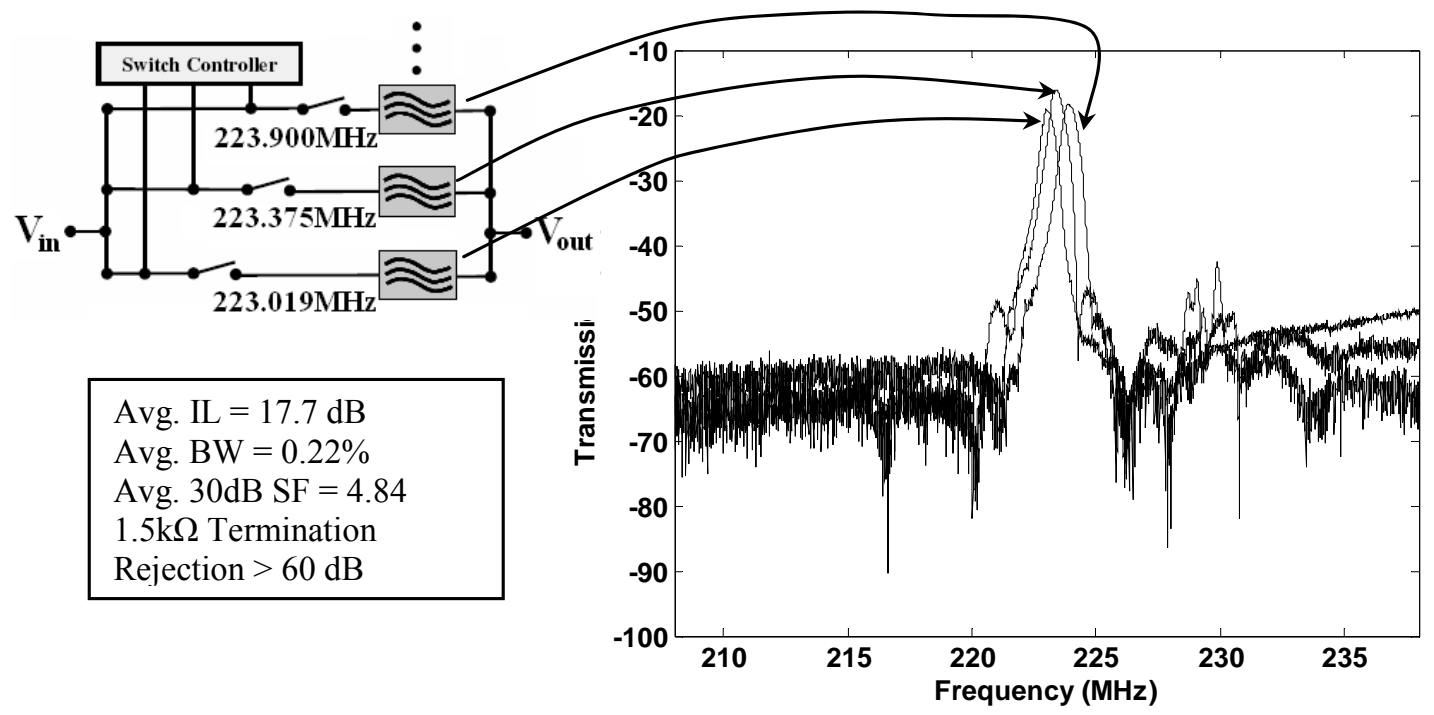

Figure 16: Experimental results for three closely spaced (in frequency) $3^{\text {rd }}$ order narrow band filters implemented using two port higher order contour-mode resonators.

Similar filters (centered at $220 \mathrm{MHz}$ ) were tested at elevated temperature, up to $400 \mathrm{~K}$. The filter shows a TCF of approximately $-27 \mathrm{ppm} / \mathrm{K}$ between 300 and $400 \mathrm{~K}$. 


\section{COMPARISON OF ONE AND TWO PORT CONTOUR-MODE RESONATORS}

This section offers a direct comparison of the one and two port contour-mode designs that were presented in the paper. Table III summarizes the key aspects that characterize each of the two designs in relation to their fabrication and performance as individual resonators or arrayed to form filters.

The fabrication process is the same for both types of devices, although variations, which require an additional mask [20], can be implemented for the two port resonator. Despite what was specifically reported in this paper, the same Figure of Merit (FOM) can be obtained from both designs and generally ranges between 5 and 30 as attested by previous work $[6,11,12]$. It has to be pointed out that the two port design is potentially subject to the excitation of spurious unwanted modes. The asymmetric excitation can generate flexural modes either in the lateral or vertical directions that can superimpose on top of the fundamental mode response and degrade the device performance (additional energy lost in unwanted modes of vibration). Instead a key advantage of the two port design is the possibility to support both single-ended and differential signals. This characteristic is unique of two port devices such as SAW and some electrostaticallydriven MEMS resonators and is not encountered in devices such as FBAR. This capability translates in the possibility to improve signal to noise ratios in receivers and also reduces the number of external components, such as baluns, that are normally required to interface single-ended signal to differential devices. An effective limitation encountered with two port devices has been the capability to expand its frequency of 
operation beyond $230 \mathrm{MHz}$. Although high frequency devices have not yet been demonstrated, there are no theoretical limitations to the frequency of operation of two port devices and the authors believe that they will soon be realized.

Table III: Comparison of key parameters between one and two port AlN contour-mode resonators presented in this work and $[6,19,20]$

\begin{tabular}{|l|c|c|}
\hline Parameter & One Port Resonator & Two Port Resonator \\
\hline Fabrication Process & Simple & $\begin{array}{c}\text { Simple, but may require } \\
\text { extra step in some } \\
\text { implementation }\end{array}$ \\
\hline FOM $\left(\mathrm{k}_{\mathrm{t}}^{2} \cdot Q\right)$ & $5-30$ & $5-30$ \\
\hline Spurious Response & $\begin{array}{c}\text { Controlled by electrode } \\
\text { patterning }\end{array}$ & $\begin{array}{c}\text { Subject to other modes of } \\
\text { vibrations }\end{array}$ \\
\hline Capacitive Feedthrough & Intrinsic device capacitance & Low $(10 \mathrm{~s} \mathrm{fF})$ \\
\hline Signal Processing & Single-Ended & $\begin{array}{c}\text { Single-Ended and } \\
\text { Differential }\end{array}$ \\
\hline $\begin{array}{l}\text { Demonstrated Frequency } \\
\text { Range }\end{array}$ & $10-803 \mathrm{MHz}$ & $20-220 \mathrm{MHz}$ \\
\hline $\begin{array}{l}\text { Filter BW (without external } \\
\text { components) in electrically } \\
\text { coupled filters }\end{array}$ & $\sim \mathrm{k}_{\mathrm{t}}{ }^{2}$ & $<\mathrm{k}_{\mathrm{t}}{ }^{2}$ \\
\hline $\begin{array}{l}\text { Out-of-band Filter } \\
\text { Rejection }\end{array}$ & $\begin{array}{c}\text { High }(30-50 \mathrm{~dB}), \text { higher in } \\
\text { differential filters }\end{array}$ & Higher $(50-70 \mathrm{~dB})$ \\
\hline
\end{tabular}

An important comparison between the two different designs can be made in relation to their use for filtering applications. Ladder filters using solely one port resonators have been demonstrated [13] and show that their BW is closely approximated by the $k_{t}^{2}$ of the material. Ladder filters using two port devices, such as the one of this paper, instead, can easily achieve BW well below $k_{t}^{2}$ without the need for any additional external component. Therefore, two port devices are better suited for narrow band filtering. Furthermore, filters implemented with two port resonators reduce relative frequency matching tolerances, since a single frequency device is required in the same filter array. 
Higher out-of-band rejection can be obtained in filters made out of two port devices since better isolation between input and output can be achieved. Similar rejection levels can be achieved in filters with one port resonators if a differential lattice structure is implemented, but would require the use of a larger number of devices. Better close-in rejection can be achieved with one port resonators thanks to the anti-resonance zeros available in the filter transfer function.

\section{SIGNAL PROCESSING VIA VIBRATING RF MEMS}

As already stated, AlN contour-mode resonators can have a tremendous impact on the wireless industry by providing smaller size and lower power RF components that can directly replace bulky and unintegrable (non silicon based) legacy technologies such as SAW and quartz crystals. Significant performance gains are to be achieved by large scale integration of multiple frequency micromechanical devices on the same substrate. By massively arraying banks of closely spaced (in frequency) band pass filters it is possible to easily implement frequency hopping spread spectrum (FHSS) transceivers (Fig. 17). This MEMS implementation will go beyond currently available FHSS transmission standards by spanning ultra wide bandwidth (as wide as $1 \mathrm{GHz}$ ) and occupying a very small board area (would instead occupy an impractically large area with current multi-package SAW or FBAR technology). This solution will provide improved signal to noise ratio and lower power consumption levels and at the same time will increase transmission capacity and security. 


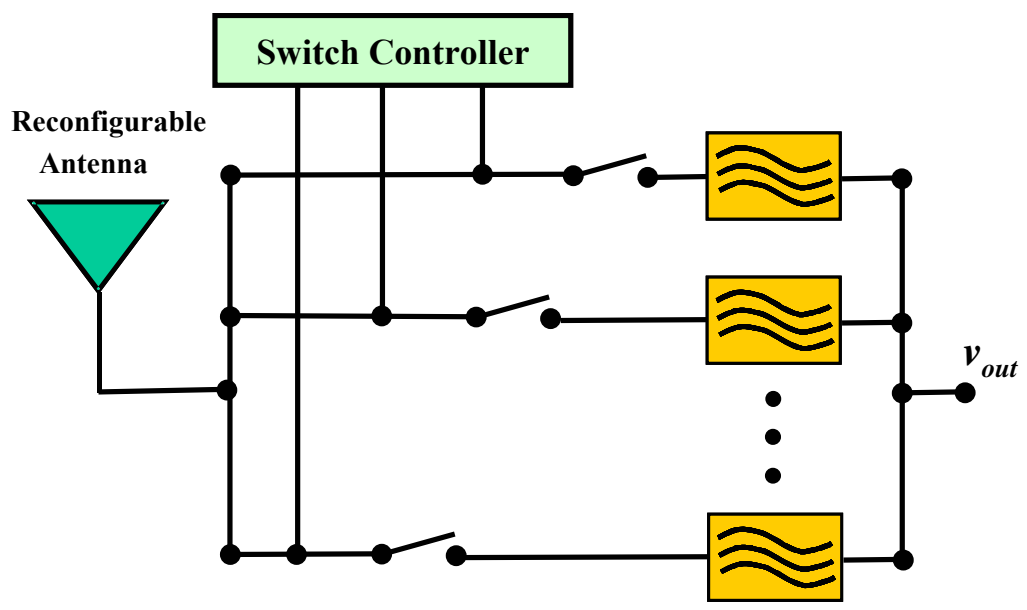

Figure 17: Next generation single-Chip RF front-ends that use closely spaced filter banks. Frequency hopping spread spectrum communication will be possible over a broad range of frequency.

Narrow bandwidth filters can also be employed for direct frequency synthesis in spread spectrum applications. Instead of using power-hungry tunable Phase Locked Loop (PLL) synthesizers, frequency reference signals are produced by generating a train of pulses from a low-noise micromechanical source and selecting among signals processed through banks of filters (Fig. 18). In this manner, faster switching speed and lower power consumption level than using indirect frequency synthesis methods based on PLL can be achieved. Direct frequency synthesis could also be pursued more aggressively by using switched banks of high $Q(Q \geq 10,000)$ and high frequency resonators eliminating the need for a low noise reference source and overall occupying a smaller chip area. This will require a significant $Q$ improvement in the demonstrated resonators and is a topic of ongoing research. 
(a)
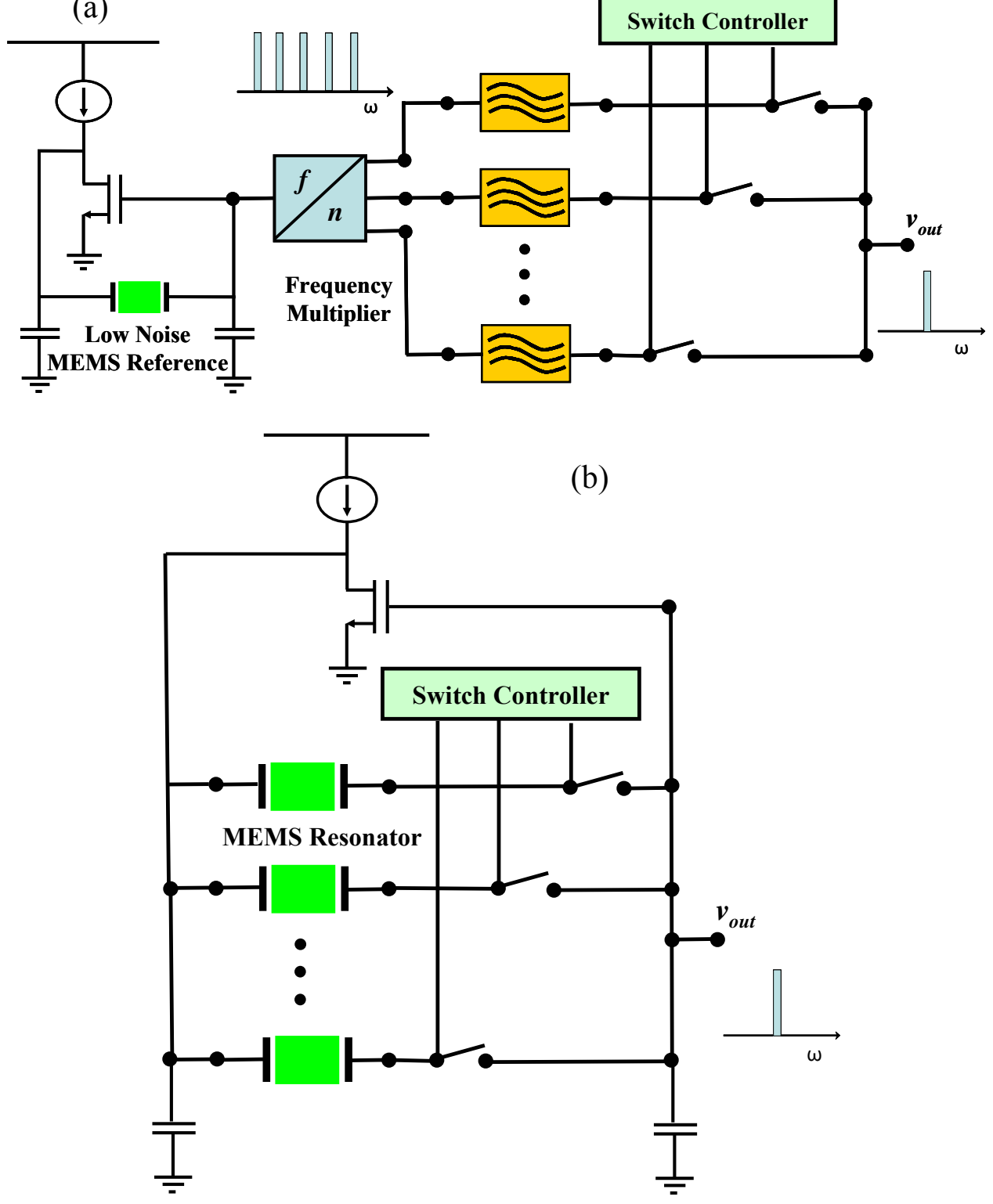

Figure 18: (a) Direct frequency synthesis via array of switched narrow band filters. A low noise MEMS reference source is used to generate a single-tone signal. A train of pulses is then produced and processed by a bank of filters. (b) Direct frequency synthesis using an array of switched high- $Q$ micromechanical resonators.

\section{CONCLUSION}

Design, fabrication and testing of novel one and two port contour-mode resonators were reported. These devices will enable new methods of frequency synthesis in spread spectrum communications. Experimental results for a new class of higher order contour- 
mode resonators were presented. Both TFE and LFE excitation were demonstrated in one port higher order contour-mode devices. The one port devices have shown frequencies as high as $803 \mathrm{MHz}$ with motional resistance of approximately $24 \Omega$. The two port devices have been employed to demonstrate banks of narrow band filters around $223 \mathrm{MHz}$. Advantages and disadvantages of one and two port devices in terms of mechanical robustness and electrical detection have been highlighted. It should be noted that each of these configurations offer specific pros and cons that must be weighed to assess their suitability for a particular application. Future work will look at improving the quality factor of these devices and increasing the frequency of operation of the two classes of resonators into $\mathrm{GHz}$ frequencies.

\section{ACKNOWLEDGMENT}

Gianluca Piazza would like to thank Chengjie Zuo, Nipun Sinha, Carlos Perez and Dr. Rashed Mahameed for their help in the fabrication and testing of some of the devices presented in the paper.

The authors also thank the staff of the Microfabrication Facilities at the University of Pennsylvania and the University of California at Berkeley for their help in the fabrication of the devices.

\section{REFERENCES}

[1] H. Chandrahalim, D. Weinstein, and S. A. Bhave, "Thickness shear mode vibrations in silicon bar resonators," Ultrasonics Symposium, 2005 IEEE pp. 898901, 2005. 
[2] M. Dubois, C. Billard, C. Muller, G. Parat, and P. Vincent, "Integration of high-Q BAW resonators and filters above IC," Solid-State Circuits Conference, ISSCC 2005, pp. 392-394, 2005.

[3] W. Jing, J. E. Butler, T. Feygelson, and C.-C. Nguyen, "1.51-GHz nanocrystalline diamond micromechanical disk resonator with material-mismatched isolating support," 17th IEEE International Conference on Micro Electro Mechanical Systems, MEMS 2004, pp.641-4, 2004.

[4] W. Jing, Z. Ren, and C.-C. Nguyen, "1.156-GHz self-aligned vibrating micromechanical disk resonator," IEEE Transactions on Ultrasonics Ferroelectrics \& Frequency Control, vol. 51, pp. 1607-28, 2004.

[5] K. M. Lakin, "A review of thin-film resonator technology," IEEE Microwave Magazine, vol. 4, pp. 61-7, 2003.

[6] G. Piazza, P. J. Stephanou, and A. P. Pisano, "Piezoelectric Aluminum Nitride Vibrating Contour-Mode MEMS Resonators," Microelectromechanical Systems, Journal of, vol. 15, pp. 1406-1418, 2006.

[7] S. Pourkamali, G. K. Ho, and F. Ayazi, "Vertical capacitive SiBARs," 18th IEEE International Conference on Micro Electro Mechanical Systems, MEMS 2005, pp. 211-214, 2005.

[8] R. Ruby and P. Merchant, "Micromachined thin film bulk acoustic resonators," Proceedings of the 1994 IEEE International Frequency Control Symposium, pp.135-8, 1994.

[9] D. Weinstein, H. Chandrahalim, L. F. Cheow, and S. A. Bhave, "Dielectrically Transduced Single-Ended to Differential MEMS Filter," 2006 IEEE, International Solid-State Circuits Conference, ISSCC 2006, pp. 318-319, 2006.

[10] L. Yu-Wei, L. Sheng-Shian, X. Yuan, R. Zeying, and C. T. C. Nguyen, "Vibrating micromechanical resonators with solid dielectric capacitive transducer gaps," Proceedings of the 2005 IEEE International Frequency Control Symposium and Exposition, pp. 128-134, 2005.

[11] G. Piazza, P. J. Stephanou, and A. P. Pisano, "Aluminum Nitride Contour-Mode Vibrating RF MEMS," Microwave Symposium Digest, 2006, IEEE MTT-S International, pp. 664-667, 2006.

[12] G. Piazza, P. J. Stephanou, J. M. Porter, M. B. J. Wijesundara, and A. P. Pisano, "Low motional resistance ring-shaped contour-mode aluminum nitride piezoelectric micromechanical resonators for UHF applications," 18th IEEE International Conference on Micro Electro Mechanical Systems, MEMS 200., pp. 20-23, 2005.

[13] G. Piazza, P. J. Stephanou, M. B. J. Wijesundara, and A. P. Pisano, "Single-chip multiple-frequency filters based on contour-mode aluminum nitride piezoelectric micromechanical resonators," The 13th International Conference on Solid-State Sensors, Actuators and Microsystems, TRANSDUCERS '05, pp. 2065-2068 Vol. 2, 2005.

[14] P. J. Stephanou, G. Piazza, C. D. White, M. B. J. Wijesundara, and A. P. Pisano, "Mechanically Coupled Contour Mode Piezoelectric Aluminum Nitride MEMS Filters," 19th IEEE International Conference on Micro Electro Mechanical Systems, MEMS 2006, pp. 906-909, 2006. 
[15] P. J. Stephanou and A. P. Pisano, "GHz Contour Extensional Mode Aluminum Nitride MEMS Resonators," to be published in Ultrasonics Symposium, 2006.

[16] K. M. Lakin, "Equivalent circuit modeling of stacked crystal filters," Proceedings of the Thirty Fifth Annual Frequency Control Symposium, pp.257-62, 1981.

[17] W. G. Cady, Piezoelectricity; an introduction to the theory and applications of electromechanical phenomena in crystals. New York: McGraw-Hill Book Company, Inc., 1946.

[18] D. P. Morgan, Surface-Wave Devices for Signal Processing. New York: Elsevier, 1991.

[19] G. Piazza and A. P. Pisano, "Dry-Released Post-CMOS Compatible ContourMode Aluminum Nitride Micromechanical Resonators for VHF Applications," 2004 Solid-State Sensor, Actuator and Microsystems Workshop, Hilton Head 2004, pp. 37-40, 2004.

[20] G. Piazza and A. P. Pisano, "Two-Port Stacked Contour-Mode Aluminum Nitride Piezoelectric Micromechanical Resonators," Eurosensors XIX, 2005.

[21] A. I. Zverev, Handbook of Filter Synthesis. New York: John Wiley and Sons, Inc., 1967.

[22] R. Ruby, P. Bradley, D. Clark, D. Feld, T. Jamneala, and W. Kun, "Acoustic FBAR for filters, duplexers and front end modules," 2004 IEEE MTT-S International Microwave Symposium Digest, vol. 2, pp.931-4, 2004.

[23] P. J. Stephanou and A. P. Pisano, "800 MHz Low Motional Resistance CountourExtensional Aluminum Nitride Micromechanical Resonators," 2006 Solid-State Sensor, Actuator and Microsystems Workshop, Hilton Head 2006, pp. 60-61, 2006. 